Case Report

\title{
Macroscopic Aspergillus Infection at the Anastomosis of a Lung Transplant Recipient
}

Shalika B. Katugaha *, Amit K. Mahajan, Meg Fregoso, Haresh Mani, Oksana A. Shlobin

Inova Fairfax Hospital, Falls Church, VA, US; E-Mails: skb1078@gmail.com; bmahaj@gmail.com; Margaret.Fregoso@inova.org; Haresh.Mani@inova.org; Oksana.Shlobin@inova.org

* Correspondence: Shalika B. Katugaha; E-Mail: skb1078@gmail.com

Academic Editor: Kamyar Afshar

Special Issue: Infections in Lung Transplantation

OBM Transplantation

2021, volume 5, issue 3

doi:10.21926/obm.transplant.2103147
Received: October 25, 2020

Accepted: June 27, 2021

Published: July 03, 2021

\begin{abstract}
A 65-year-old female with a history of right lung transplantation (LTX) for chronic obstructive pulmonary disease (COPD) 9-years prior complicated by grade 3 bronchiolitis obliterans syndrome (BOS) was admitted to the hospital for chest pain and dyspnea on exertion (DOE). Her bronchoscopy revealed fluffy, endobronchial lesions that were adherent to the right sided anastomosis resulting in mild obstruction of the right mainstem bronchus. Pathology from biopsies of the endobronchial lesions revealed hyphal fungal organisms consistent with Aspergillus species. Following antifungal therapy with voriconazole, her follow up bronchoscopy 37 days post the original bronchoscopy revealed resolution of the endobronchial lesions. The patient's case is unique in: presentation 9 years posttransplantation, the absence of prior Aspergillus colonization, and the appearance of the endobronchial lesions. Following Ltx, immunosuppression in combination with allograft exposure to the environment increases the risk of opportunistic infections, especially saprophytic infections including Aspergillus.
\end{abstract}

\section{Keywords}

Aspergillus; fungal infection; lung transplant; endobronchial, anastomosis

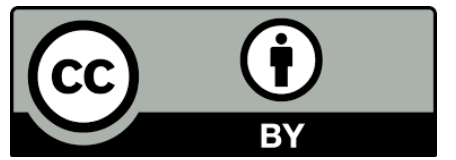

(c) 2021 by the author. This is an open access article distributed under the conditions of the Creative Commons by Attribution License, which permits unrestricted use, distribution, and reproduction in any medium or format, provided the original work is correctly cited. 


\section{Description}

A 65-year-old female with a history of right lung transplantation (LTX) for chronic obstructive pulmonary disease (COPD) 9-years prior complicated by grade 3 bronchiolitis obliterans syndrome (BOS) was admitted to the hospital for chest pain and dyspnea on exertion (DOE). The patient noted persistent sharp chest pain below her sternum along with progressive DOE over the past three weeks. No fevers or chills were reported. The patient also had a history of steroid induced diabetes mellitus, hypertension, chronic kidney disease, history of melanoma, and gastroesophageal reflux disease. She was on decreased immunosuppression due to history of melanoma: tacrolimus $1.5 \mathrm{mg}$ in the morning and $2 \mathrm{mg}$ in the evening (trough goal $~ 5$ ) and prednisone at $5 \mathrm{mg}$ daily.

Following negative cardiac workup and unrevealing chest computerized tomography (CT) scan, the patient underwent flexible bronchoscopy to assess for possible infectious and immunologic etiologies explaining her symptoms. The airway exam revealed fluffy, endobronchial lesions that were adherent to the right sided anastomosis resulting in mild obstruction of the right mainstem bronchus [Figure 1]. Prolene (standard) sutures used for the airway anastomosis were present. There was no unusual appearance to the sutures, and in our experience, we always see at least some parts of the anastomotic sutures throughout the life of the graft. No other lesions were noted throughout the bilateral tracheobronchial tree. The flexible forceps were used to easily debride and completely remove the lesions. Post removal, the base of the lesion appeared clean with visually intact wall. A bronchoalveolar lavage (BAL) and transbronchial forceps biopsies (TBBx) of the allograft were also performed.

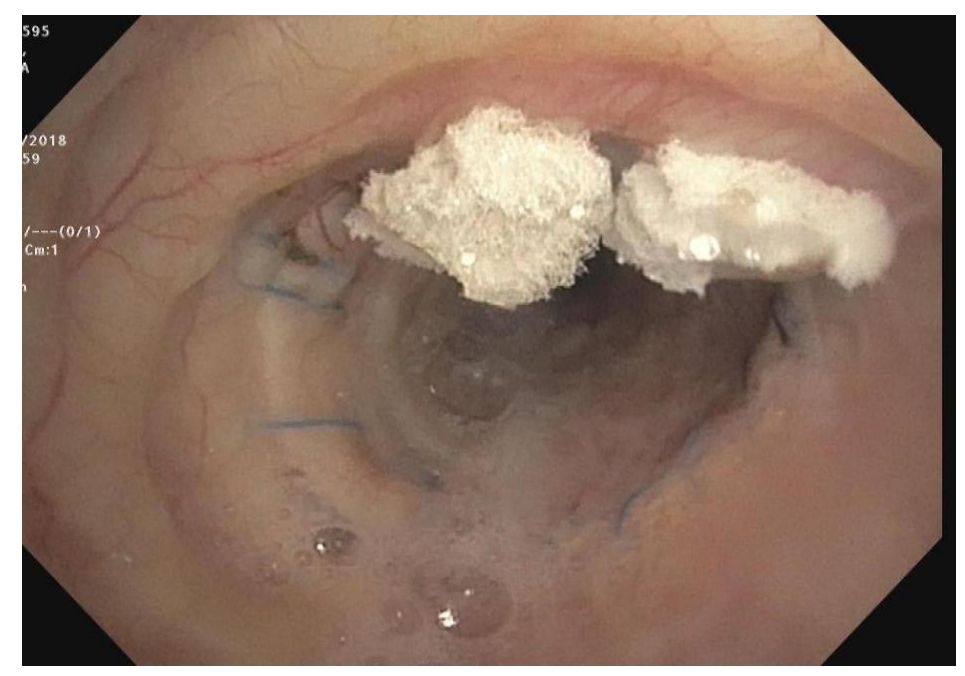

Figure 1 Bronchoscopy exam revealed fluffy, endobronchial lesions that were adherent to the right sided anastomosis resulting in mild obstruction of the right mainstem bronchus.

Pathology results from biopsies of the endobronchial lesions revealed hyphal fungal organisms consistent with Aspergillus species [Figure 2]. Allograft BAL fungal cultures were finalized as negative at 6 weeks, and allograft revealed no evidence of rejection. Her beta-D-glucan assay was negative. The patient was started on intravenous voriconazole and amphotericin nebulizers and 
transitioned to oral voriconazole upon discharge. Though there are no guidelines for anastomotic Aspergillus infection, voriconazole was selected as it is the treatment of choice for invasive aspergillosis. She was already on decreased immunosuppression, so her immunosuppression regimen was not changed.

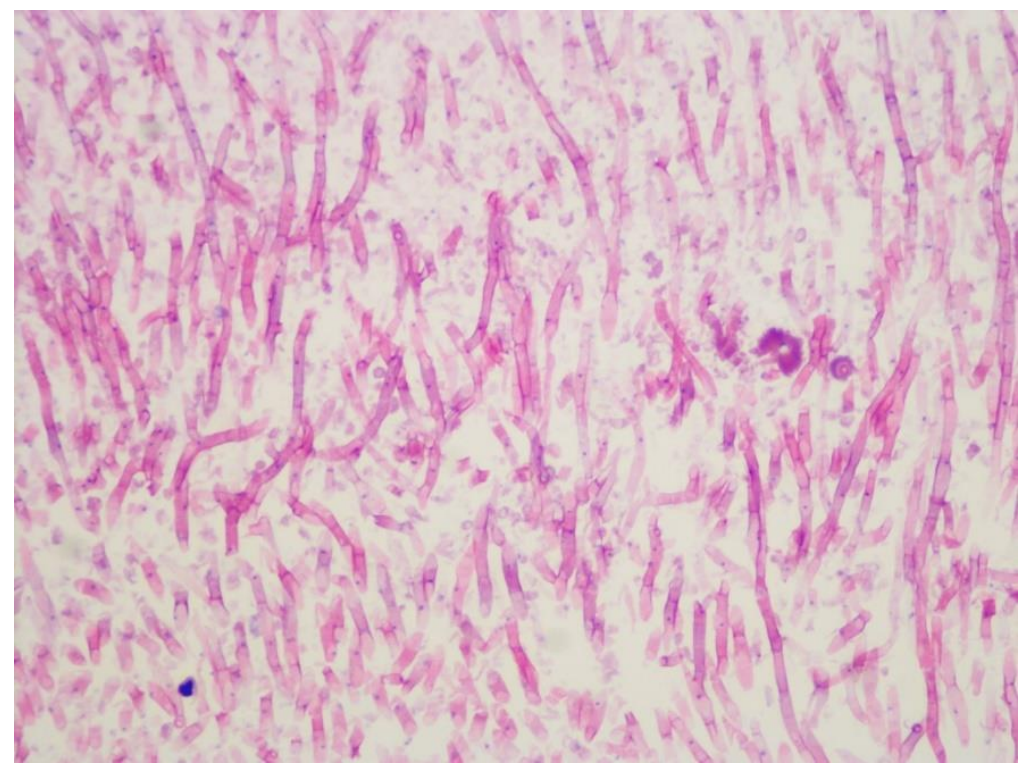

Figure 2 Pathology results from biopsies of the endobronchial lesions revealed hyphal fungal organisms consistent with Aspergillus species.

She had a follow up bronchoscopy 37 days post original bronchoscopy which revealed resolution of the endobronchial lesions [Figure 3]. The following day, the patient collapsed at home, was taken to the local hospital, and passed away the same day. The family did not request autopsy.

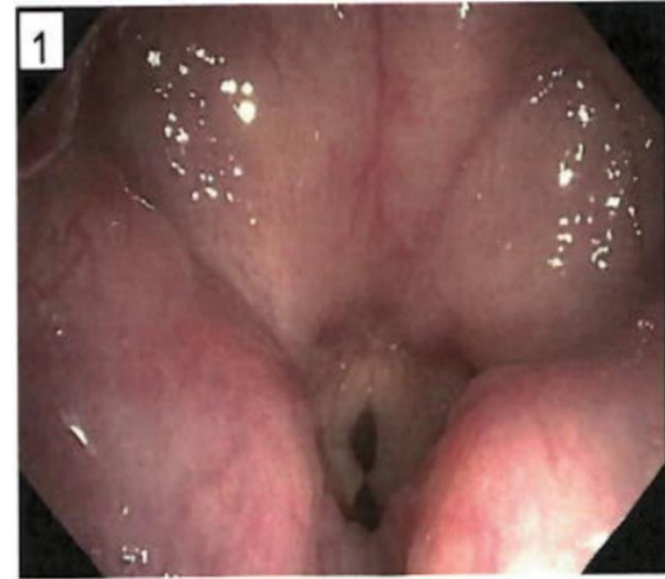

Vocal cords

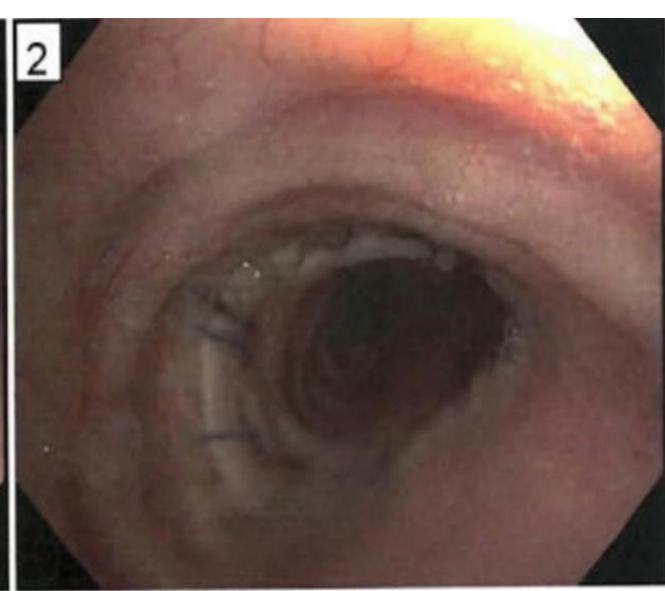

Right sided anastomosis

Figure 3 Bronchoscopy exam 37 days following original debridement bronchoscopy and oral voriconazole.

Following Ltx, immunosuppression in combination with allograft exposure to the environment increases the risk of opportunistic infections, especially saprophytic infections. Saphrophytic Aspergillus infection involving bronchial anastomoses has been described in lung transplant 
recipients [1]. In Nunley and colleagues' series of fifteen saprophytic fungal infections involving the bronchial anastomosis in Ltx recipients, the incidence of saprhophytic fungal infection of the bronchial anastomosis was as high as $24.6 \%$, with Aspergillus species being responsible for two thirds of the cases [2]. In another report, Aspergillus fumigatus was responsible for almost $90 \%$ of all tracheobronchial infection in Ltx recipients [1]. The median time from transplantation to diagnosis of saprophytic fungal infection was 35 (range 13-159) days [2]. According to the review by Singh, the median time from transplantation to diagnosis of Aspergillus bronchial anastomotic infection was 2.7 months [3]. In a retrospective study of 86 Ltx recipients with Aspergillus infection, all bronchial anastomotic infections occurred within 3 months of transplantation [3]. In all of these series, fungal endobronchial infections occurred during the first three months when immunosuppression is typically maximal. Our patient's presentation at 9 years post-transplant and on reduced immunosuppression is atypical.

The patient's predisposing factors for endobronchial Aspergillus infection include lung transplant, immunosuppressive medications, and diabetes mellitus [4]. The patient also had visible sutures. In patients treated with resectional surgery, bronchial stump aspergillosis results from fungal colonization of the suture material [5]. It has been suggested that risk of stump aspergillosis can be decreased by using an unbraided nylon monofilament rather than silk sutures [4]. Another risk factor for endobronchial Aspergillus infection is airway colonization with Aspergillus [1]; our patient was not colonized, evidenced by several negative past bronchoscopies.

The majority of Aspergillus-related tracheobronchial lesions in Ltx recipients occurred in the transplanted lung and involved the anastomosis line [1], as in our patient. In Ltx recipients, bronchoscopic appearance of Aspergillus includes pseudomembrane formation, bronchial stenosis in the anastomosis line, ulcerations, and black eschar [1], rendering unique the fluffy endobronchial lesions in our patient. Mortality rate calculated from 40 studies was $23.7 \%$ in patients with Aspergillus tracheobronchitis and/or anastomotic infection versus $81.8 \%$ and $50 \%$ in patients with invasive pulmonary aspergillosis and disseminated infections [1]. We were unable to determine the patient's cause of death, but it was unlikely due to fungal infection based on acute presentation.

While the delineation of fungal infection of the surgical anastomosis versus fungal infection of the allograft may be challenging, this case provides an example of a macroscopic Aspergillus infection at the anastomosis in a lung transplant recipient. This case denotes the importance of bronchoscopy in the diagnosis and management of fungal endobronchial disease in lung transplant recipients.

\section{Learning points/take home messages}

- Following lung transplantation, immunosuppression in combination with allograft exposure to the environment increases the risk of opportunistic infections, especially saprophytic infections. Saphrophytic Aspergillus infection involving bronchial anastomoses has been described in lung transplant recipients [1].

- While the delineation of fungal infection of the surgical anastomosis versus fungal infection of the allograft may be challenging, this case provides an example of a macroscopic Aspergillus infection at the anastomosis in a lung transplant recipient.

- This case denotes the importance of bronchoscopy in the diagnosis and management of fungal endobronchial disease in lung transplant recipients. 


\section{Author Contributions}

All of the authors participated in the care of the patient. Shalika Katugaha and Amit Mahajan wrote the article. Meg Fregoso outlined details about the case. Haresh Mani provided the pathology pictures. Oksana Shlobin took the bronchoscopy pictures. Meg Fregoso, Haresh Mani, and Oksana Shlobin edited the case report.

\section{Competing Interests}

The authors have declared that no competing interests exist.

\section{References}

1. Krenke R, Grabczak E. Tracheobronchial manifestations of Aspergillus infections. Sci World J. 2011; 11: 2310-2329.

2. Nunley DR, Gal AA, Vega JD, Perlino C, Smith P, Lawrence EC. Saprophytic fungal infections and complications involving the bronchial anastomosis following human lung transplantation. Chest. 2002; 122: 1185-1191.

3. Singh N, Husain S. Aspergillus infections after lung transplantation: Clinical differences in type of transplant and implications for management. J Heart Lung Transplant. 2003; 22: 258-266.

4. Karnak D, Avery RK, Gildea TR, Sahoo D, Mehta AC, et al. Endobronchial fungal diseases: An under-recognized entity. Respiration. 2007; 74: 88-104.

5. Sole A, Morant P, Salavert M, Pemán J, Morales P, the Valencia Lung Transplant Group. Aspergillus infections in lung transplant recipients: Risk factors and outcome. Clin Microbiol Infect. 2005; 11: 359-365.

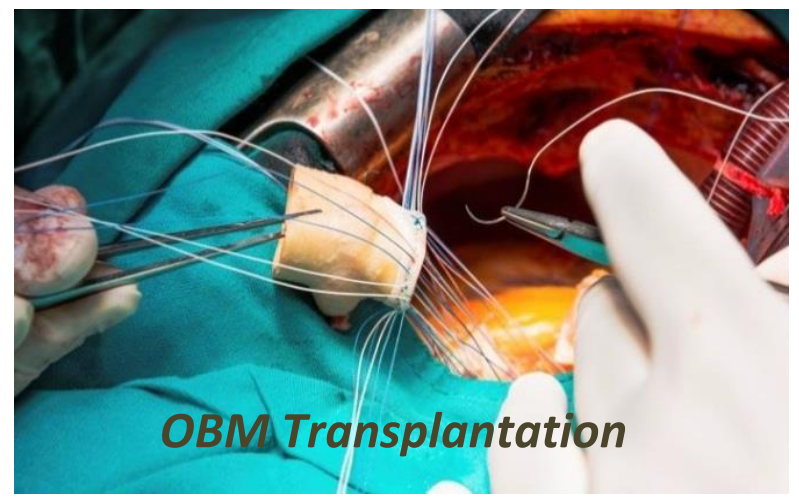

Enjoy OBM Transplantation by:

1. Submitting a manuscript

2. Joining in volunteer reviewer bank

3. Joining Editorial Board

4. Guest editing a special issue

For more details, please visit:

http://www.lidsen.com/journals/transplantation 